Cuadernos de Filología Clásica. Estudios Latinos

ISSN: 1131-9062

http://dx.doi.org/10.5209/cfcl.73006

\title{
Análisis del prólogo al Libro II de la Historia Francorum de Gregorio de Tours: su sentido programático
}

\author{
Francisco Javier Tovar Paz ${ }^{1}$
}

Recibido: 11 de septiembre de 2020 / Aceptado: 30 de noviembre de 2020

Resumen: El estudio ofrece un análisis del prólogo al Libro II de la Historia Francorum de Gregorio de Tours a partir del proyecto historiográfico que el propio texto propugna. En la alternancia e imbricación entre motivos religiosos, políticos y cronológicos, dicho prólogo aporta las consideraciones estilísticas y de género literario que permiten interpretar la obra en su conjunto a la manera de un vademécum para los monarcas merovingios a la vez que, a partir del Libro de los Reyes del Antiguo Testamento, permite percibir la obra en su conjunto como una continuatio bíblica.

Palabras clave: Gregorio de Tours; Historia Francorum; historiografía; política merovinga; Biblia.

\section{[en] Analysis of the prologue to Book II of the Historia Francorum by Gregory of Tours: its programmatic sense}

\begin{abstract}
The study offers an analysis of the Prologue to Book II of the Historia Francorum by Gregory of Tours based on the historiographic project that the text itself advocates. In the alternation and overlapping between religious, political and chronological motifs, this prologue provides the stylistic and literary genre considerations that allow the interpretation of the work as a whole in the manner of a vade mecum for the Merovingian monarchs while, based on the Book of Kings of the Old Testament, it allows the perception of the work as a whole as a biblical continuatio.

Keywords: Gregory of Tours; Historia Francorum; historiography; Merovingian politics; Bible.

Sumario: 1. Introducción: Una percepción holística de la Historia Francorum. 2. Sobre la estructura de la Historia Francorum en su conjunto. 3. El prólogo al libro II: sentidos general y particular de la Historia Francorum. 4. Un planteamiento historiográfico de carácter político. 5. Conclusión: La continuatio bíblica como subgénero historiográfico. Referencias bibliográficas.
\end{abstract}

Cómo citar: Tovar Paz, F. J. «Análisis del prólogo al Libro II de la Historia Francorum de Gregorio de Tours: su sentido programático», Cuad. Filol. Clás. Estud. Lat. 40.2 (2020), 231-246.

Universidad de Extremadura.

Correo eletrónico: fjtovar@unex.es. 


\section{Introducción: Una percepción holística de la Historia Francorum}

Uno de los aspectos de mayor debate académico acerca de la Historia Francorum de Gregorio de Tours radica en la comprensión de su sentido último en relación con el género literario y la idoneidad de la temática abordada a este respecto, en lo que también influye la disparidad de títulos que la tradición ha legado: Historia Francorum, Historiae o Historiarum Libri. Ello deriva en cuestiones relativas a si responde la obra a la intención de redactar una historia de la Iglesia; o si se trata de una historia de las disputas entre los reinos y monarcas que suceden al Imperio Romano; o de una historia más específica centrada en las distintas facciones del pueblo Franco una vez instalado en la antigua Galia romana; o, en fin, de una reivindicación del papel de la ciudad de Tours y de su obispado en la política merovingia. La respuesta procede probablemente de la suma de unas propuestas que abarcan desde la general a la local (de acuerdo con la importante, aunque ya lejana en el tiempo, aportación de Oldoni 1972; también Breukelaar 1994, Murray 2005 y Heinzelmann 2001 se presentan como relevantes hitos académicos contemporáneos, en unos momentos de renovado interés por la figura del Turonense) y, sobre todo, a partir de una síntesis que nace de la voluntad personal del historiador de llevar a cabo una obra de calado temporal universal al tiempo que contemporánea (según se postula en Wood 1994 y Mitchell \& Wood 2002). No obstante, no faltan propuestas de interpretación que denuncian el debate "poder celestial-poder terrenal" como motor exclusivo de la historiografía cristiana, a cambio de su papel como intérpretes tanto de los textos como de los hechos, sin que se planteara rivalidad con los monarcas; o, en otras palabras, que la singularidad de historiografía cristiana no se concibe en la idea de que la jerarquía eclesiástica se postule para sustituir al poder civil (Werner, 1987); y sin que falten tampoco claves de lectura que alejan del texto una voluntad historiográfica expresa en su sentido tradicional por parte del Turonense a cambio de una finalidad más instruccional y catequética (Carozzi 2007).

En otras palabras, la obra surge de la combinación de diferentes subgéneros históricos, que van desde la lectura de la Biblia en su conjunto o de alguno de sus libros como texto histórico hasta la crónica de carácter esquemático; desde la inserción de cuentos bajo la forma de anécdotas o relatos novelescos hasta la aportación de documentos de índole jurídico-política (Martínez Pizarro 2016) y la presencia del historiador en su texto incluso como profeta (Reimitz 2015; también Heinzelmann 2001, 41-48).

De todo ello se desprende, ciertamente, un conjunto desigual, que provoca que la obra parezca responder a una estructura en apariencia descompensada en la que la presencia del escritor es fundamental, y ello a pesar de que el texto se presenta como un relato histórico que escapa del control de una única persona (Adamik 2012), y en el que es posible apreciar, de acuerdo con Heinzelmann, cómo el autor equipara el papel de los poderes religioso y civil, es decir, de la Iglesia y la monarquía goda (Heinzelmann 2001, 153 y 160-172). Al tiempo y de manera harto probable, su redacción, que fue de larga gestación y duración, varía en función de los intereses del escritor a lo largo del tiempo (Banniard 1978). Pero el propio historiador es consciente de los cambios de tono en fondo y forma y, a pesar de todo, propugna en su cierre una lectura compacta del texto, como si, al margen de las partes y tonos diferentes, existiera una voluntad deliberada de la forma final que quiere presentar como conjunto; de ahí también la importancia que los estudios conceden desde siempre al denominado testamento de Gregorio en Historia Francorum 10.31. 
En efecto, aunque resulta difícil conocer en detalle el proceso de elaboración del texto (Murray 2008), sí es posible constatar cómo la obra nace de una doble voluntad: en primer lugar: la voluntad de llevar a cabo una especie de continuatio bíblica, de forma que se diera cabida desde los libros históricos del Nuevo Testamento hasta la época del historiador, pues, según hemos apuntado ya, el mismo Turonense llega a ser percibido como profeta; y, en segundo lugar: la voluntad de intervenir sobre la política contemporánea desde sus márgenes, es decir, desde una posición exterior a las decisiones y sucesos políticos, aunque con una evidente voluntad de influir sobre estos (Heinzelmann 2001; Tovar 2011 o Murray 2015). Ello deriva en que se anteponga lo expresamente religioso sobre lo civil (o, como mínimo, uno y otro se dan en un plano de igualdad) en un contexto en el que la formación cultural está asociada al cristianismo católico y, por ende, depende de la población tardorromana sometida; así, la actuación política se deja en manos de los reyes merovingios, los cuales, en ocasiones, dan cabida a otros credos cristianos, como la fe arriana. En otras palabras, se trata de una forma implícita de influir de manera eficaz y reivindicativa sobre las cortes merovingias desde un reconocimiento explícito de su jerarquía sobre los dominios de la antigua Galia, a la vez que desde su dependencia religiosa de los estamentos tardorromanos aún presentes en la sociedad de la época (Croke 2003).

De esta forma, si bien la disposición cronológica de la obra parece precisa en su continuidad, la estructura no deja de ofrecer cierta complejidad compositiva, con continuas variaciones y cambios de orientación, que pueden ser simultáneos o aparentemente aleatorios, dispuestos además a través de una serie de prólogos que afectan no a todos, sino a determinados libros y contenidos, según es práctica del propio escritor a la hora de presentar otros textos (Janson 1964).

\section{Sobre la estructura de la Historia Francorum en su conjunto}

En efecto, la Historia Francorum cuenta con un prefacio general y con distintos prólogos internos que abren capítulos diferentes a lo largo de la disposición cronológica de la obra: se trata de prólogos que merecen una atención específica. De hecho, su disposición, más que irregular o aleatoria, deviene estratégica al respecto de los límites temporales sobre los que tales prólogos anuncian su influencia. Por lo demás, según apreciaremos más en detalle, cada prólogo aborda unos contenidos singulares, unos temas específicos que condicionan la lectura de los libros y capítulos que introducen. Tales prólogos se presentan como introducciones metahistoriográficas, pues sirven al autor para comentar el sentido general de los sucesos, como una especie de previsión panorámica, pero también se utilizan para desgajar lo propiamente historiográfico de lo hagiográfico (Thürlemann 1974). De ahí la importancia de establecer pares temáticos que se impone el historiador a sí mismo en la presentación de sus textos, e imbricar de esta forma contenidos que podrían ser hagiográficos (como en otras obras del escritor) en un contexto histórico. También es cierto que los contenidos hagiográficos, que se presentan como una incumbencia singular de la Iglesia, tienen un uso político; de ahí que los límites a veces resulten difusos, como es preciso testimonio la Vita Desiderii (Vita vel passio Sancti Desiderii) del rey visigodo Sisebuto (Fontaine 1980). 
Tales prólogos se corresponden con las cuatro etapas cronológicas en torno a las que se organiza el texto ${ }^{2}$. A tales cuatro prólogos se han de añadir como pasajes equiparables el Prefacio General que abre el Libro I y el Epílogo que cierra la obra tras el Libro X. Así, es posible desglosar la obra de acuerdo con la siguiente estructura:

1. Libro I: abarca desde el Génesis bíblico a la figura de san Martín de Tours, ya en momentos de Imperio Romano avanzado (siglo IV). Por consiguiente, en el libro se concentran dos épocas de cariz muy distinto, la época bíblica y la época imperial romana o, con más precisión, postevangélica, con las amenazas heréticas que caracterizan esta última etapa; se trata de un libro que se abre con el Prefacio General de la obra.

2. Libros II al IV: afecta a los siglos V y VI (una centuria y prácticamente tres cuartas partes de la siguiente), representados por dos reinados fundamentales, los de Clodoveo y Sigiberto. Los libros segundo y tercero cuentan con prólogo; lo cual es indicio importante de la singularidad que el autor quiso imprimir a las figuras de Clodoveo (primer rey cristiano y fundador de la dinastía merovingia) y de Sigiberto (uno de los monarcas de reinado más longevo, bajo el que se producen graves disputas internas que condicionan fuertemente el presente del autor).

3. Libros $\mathrm{V}$ al $\mathrm{X}$ : recogen las últimas dos décadas del siglo VI, con capítulos centrados en dos personalidades sucesivas, Chilperico y Gontrán, que representan respectivamente los polos negativo y positivo de la época de dominio franco contemporánea del escritor. Los libros V y IX cuentan con prólogo, además del $\mathrm{X}$, cuyo epílogo funciona como una especie de testamento general de la obra (Hist. 10.31).

Según se puede apreciar, el diseño de la obra ofrece un paulatino proceso de concentración cronológica a la vez que de ampliación de los temas abordados: más contenido a propósito de etapas que se van aglutinando progresivamente, o, en otras palabras, mediante libros en los que se reduce cada vez más su extensión temporal. Se trata de una opción nítidamente deliberada por parte del autor. A este respecto, los textos introductorios (prólogos) van marcando en líneas generales tales etapas, sobre todo en lo que concierne a los hitos de la Historia de los Reyes Merovingios con nombre propio: Clodoveo, Sigiberto, Chilperico y Gontrán. A tales prólogos se ha de añadir el prefacio general, que abre el libro I, y un epílogo en el libro X. En el primer libro, dedicado en su conjunto a la historia anterior a los godos, se hace especial hincapié en la figura de san Martín de Tours; por su parte, el epílogo presenta un carácter testamental en torno a la pervivencia de la obra al tiempo que se reflexiona acerca de unos estilo y estructura que resultan, al fin y al cabo, deliberados.

Tal es el contexto global en el que se entienden los temas presentes en el prefacio al conjunto de la obra: la decadencia de la cultura bajo la etapa histórico-política de los monarcas godos, el sufrimiento de las iglesias por los riesgos de las herejías y el peligro que corren a este respecto, $\mathrm{y}$, finalmente, la necesidad del historiador de llevar a cabo una decisión personal y su intención pragmática de salvar los hechos descritos para el futuro, además de hacerlo desde su sede en Tours (Heinzelmann 2001, Moore 2011).

Se trata de etapas desglosadas también así en todos los estudios, como, por citar incluso el caso de un análisis que rechaza la voluntad historiográfica en su sentido clásico en el Turonense, en Carozzi 2007. 
En lo que se refiere al prólogo al Libro I, que sigue directamente al prefacio general, este se organiza a partir del contraste entre la decadencia política, que degenera en bella regum, y la decadencia religiosa, que se plasma en bella martyrum; uno y otro polos convergen en bella ecclesiarum, los cuales son los que, en principio, justifican la labor de Gregorio de Tours (Wallace-Hadrill 2019). De esta forma, la mirada está puesta en el presente, y en los márgenes de los conflictos bélicos y de los cristianos de los primeros tiempos (si bien en las guerras de las Iglesias importa la lucha contra los herejes, es decir, las guerras intestinas o civiles dentro del cristianismo). El sentido del texto posee, por consiguiente, una evidente orientación pragmática, con el fin de comprender cómo los enfrentamientos que han provocado la decadencia política y la decadencia religiosa, así como la aparición de la Iglesia y de san Martín de Tours, se convierten en referente del momento coetáneo del Turonense, de esas dos últimas décadas a las que dedica la mitad de la obra. Este es el contexto en el que, aunque, como sucede con los restantes prólogos, aporta también temas propios, se sitúa el prólogo al Libro II, al que se dedica el análisis del presente estudio.

Por su parte, el prólogo del libro III se centra en el enfrentamiento entre la herejía arriana encarnada en los monarcas godos y el catolicismo de la población gobernada; se hace desde una síntesis entre tensiones sociales y culturales que, en buena medida, constituyen el marco de la obra.

El prólogo al Libro V plasma la polaridad heredada de los pasajes programáticos previos, pero lo hace en términos puramente escatológicos, pues alude al apocalipsis o destrucción de la que se hace acreedora la parte política y a la prosperidad y esplendor que merece la parte religiosa. Así, el primer polo refleja las tensiones entre el monarca y la iglesia, en tanto el segundo implica la aceptación por parte del rey de los criterios emanados desde la iglesia (Halsall 2007).

Finalmente, el último prólogo propiamente dicho, que se corresponde con el comienzo del Libro IX, ofrece una presentación esquemática, claro indicio del cambio de registro historiográfico en el que está operando Gregorio de Tours para cerrar la obra, haciéndolo hacia el subgénero de la chronica (Galán 1994). En ello influye la absoluta contemporaneidad de los contenidos propia de dicha crónica, la cual se le presenta con un carácter más neutro, meramente expositivo. La obra se cierra con un enfático epílogo que, al margen de otras consideraciones, incide en el carácter unitario del conjunto a pesar de las diferencias de las partes.

En efecto, de alguna manera, Gregorio de Tours ha ido planteando hasta el Libro $\mathrm{V}$ un crescendo de temas que avanzan desde la oposición entre Monarquía e Iglesia, las menciones al subgénero histórico del que se hace acreedor el tratamiento de un polo u otro, las figuras que los encarnan o representan, los movimientos cristianos o sectas en que se apoyan y, finalmente, los resultados alternativos, como desastre o triunfo, de acuerdo con un cuadro como el siguiente:

\begin{tabular}{|llllll|}
\hline & PREF. & PRÓL.I & PRÓL.II & PRÓL.III & PRÓL.V \\
POLÍtICA: & Reyes & Chronica & Guerreros & Arrianismo & Apocalipsis \\
RELIGIÓN: & Iglesia & Historia & Mártires & Catolicismo & Prosperidad \\
& & & & & \\
\hline
\end{tabular}


Es más, en el prólogo del Libro IX, en la parte dedicada al rey Gontrán en calidad de referente de la obra, la chronica, que se anuncia como género propio del tratamiento relativo a los reyes en el prólogo al Libro I, se convierte en una manifestación patente del estilo al que recurre el autor. De esta forma Gregorio propugna una redacción distinta, que no solo coincide con la tensión política de la contemporaneidad, sino que esta se presenta como síntesis entre las tensiones descritas, con la consiguiente desaparición de la polaridad entre política y religión, fundidas en la mera descripción de los sucesos.

\section{El prólogo al libro II: sentidos general y particular de la Historia Francorum}

Tal es el contexto en el que resulta de interés el análisis del Prólogo al Libro II (considerado con cierto detalle en Goffart 1988, y sobre todo en Heinzelmann 2001, 103 104; también en Adamik 2012). En efecto, Gregorio de Tours presenta este Libro II en la cadena temática que hace avanzar la obra con especial hincapié en la visión que se hace de guerreros y mártires tras la desaparición del Imperio Romano y tras la mostración de una Iglesia capaz de convivir con el Imperio según se sintetiza en la figura de Martín de Tours, personalidad que constituye una invocación perenne por dar origen a la sede episcopal que el historiador regenta. De hecho, el primer capítulo del Libro II se abre con la figura del obispo Bricio, sucesor de Martín de Tours, y de las posteriores persecuciones tanto políticas como heréticas que se dan dentro del ámbito cristiano, hasta llegar a la figura del rey Clodoveo, sobre el que pivota el sentido de conjunto del Libro, que se cierra con su muerte.

Ciertamente, Clodoveo -o Clovis- encarna la pervivencia de cierta continuidad del Imperio Romano en un entorno que está mutando hacia realidades histórico-políticas y culturales diferentes, como refleja la conversión al catolicismo del rey, cuya fe originaria es la arriana, tras obtener la victoria en la batalla de Tolbiac. De esta forma, la victoria bélica, y por ende política, aparece recompensada por el reconocimiento religioso a los católicos que deriva en la propia conversión del rey. La suma de claves políticas y religiosas hace que Clodoveo sea presentado como modelo del pasado para el presente al que se orienta la obra; es decir, la peripecia vital e histórica del rey aparece como lección magistral para futuros gobernantes; más aún cuando se trata del primer rey de la dinastía y cuando por vez primera se aborda la figura de un rey, algo que va a marcar una importante impronta sobre el sentido de la Historia Francorum como otro aspecto clave en la interpretación de la obra como conjunto (Meens et al. 2016).

En este contexto el prólogo al Libro II, además de hilvanar el final del mundo romano y la figura de san Martín de Tours con la emergente Iglesia gala y la administración política de los francos, ofrece otros aspectos que superan la coyuntura cronológica del Libro y permiten trascender la intención del historiador. Si bien existe traducción reciente en español (Herrera 2013), preferimos aportar nuestra versión, en la que, además, nos hacemos eco de algunas de las discusiones textuales planteadas (Adamik 2012), dice el texto (seguimos la edición clásica de Bruno Krusch, reeditada a mediados del siglo XX: Krusch \& Levinson 1951):

Prosequentes ordinem temporum, mixte confusequae tam virtutes sanctorum quam strages gentium memoramus. Non enim inrationabiliter accipi puto, se fili- 
cem beatorum vitam inter miserorum memoremus excidia, cum idem non facilitas scripturis, sed temporum series praestitit. Nam sullicitus lector, si inquirat strinue, invenit inter illas regum Israheliticorum historias sub Samuhel iustum Fineen interisse sacrilegum ac sub David, quem Fortem manu dicunt, Golian alophilum conruisse. Meminiat etiam sub Heliae eximii vatis tempore, qui pluvias cum voluit abstulit et cum libuit arentibus terris infudit, qui viduae paupertatem oratione locopletavit, quantae populorum strages fuere, quae famis vel quae siccitas miseram oppraesserit humum; quae sub Ezechie tempore, cui Deus ad vitam quindecim annos auxit, Hierusolima mala pertulerit. Sed et sub Heliseum prophetam, qui mortuos vitae restituit et alia in populis multa miracula fecit, quantae internitiones, quae miseriae ipsum Israheliticum populum oppraesserunt. Sic et Eusebius, Severus Hieronimusquae in chronicis atque Horosius et bella regum et virtutes martyrum pariter texuerunt. Ita et nos idcircum sic scripsemus, quod facilius saeculorum ordo vel annorum ratio usque nostra tempora tota repperiatur. Venientes ergo per antedictorum auctorum historias, ea quae in posterum acta sunt Domino iubente disseremus.

En traducción:

Siguiendo su orden cronológico, rememoramos mediante interpolaciones y de manera intercalada tanto las virtudes de los santos como las perversidades de los gentiles. Considero que no tiene por qué entenderse como algo ilógico, si recordamos la vida feliz de los piadosos en medio de la destrucción de los malvados cuando así lo permite no la conveniencia del historiador sino la sucesión de los tiempos. En efecto, un lector puntilloso, si lo examina con atención, encontrará que en medio de aquellas historias de los reyes de los Israelitas en tiempos del justo Samuel se intercala el sacrilegio de Fineas; y que, a manos de David, al que llaman el de poderoso puño, sucumbió Goliat el filisteo. También podría recordar que en tiempos del eximio profeta Elías, quien detenía la lluvia a voluntad y a voluntad la derramaba sobre las tierras secas y quien con su palabra tornó la pobreza de una viuda en riqueza, cuánta destrucción de naciones había, cuánta hambre y sequía asolaba la tierra maldita. O qué males acaecían sobre Jerusalén en tiempos de Exequías, a quien Dios alargó su vida en quince años. O también en tiempos del profeta Eliseo, que devolvió muertos a la vida e hizo otros muchos milagros en medio de la gente, cuántas masacres, cuántas desgracias oprimían al mismo pueblo de Israel. De igual manera, Eusebio, Severo y Jerónimo en sus respectivas “Crónicas”, también Orosio, fueron entretejiendo las guerras de los reyes al tiempo que las virtudes de los mártires. Y por ese mismo motivo nosotros lo hemos puesto así por escrito, para que con facilidad se encuentre de forma continua en un todo el orden de los siglos o la sucesión de los años hasta hoy día. Más adelante, al cabo de las historias de los autores citados y si así lo consiente la voluntad del Señor, desarrollaremos los hechos acaecidos con posterioridad.

En principio el prólogo ofrece dos contenidos, uno de fondo y otro formal. El tema de fondo se refiere a la convivencia entre situaciones y figuras en virtud de su carácter positivo y negativo: las primeras situaciones son las cristianas, las segundas, las paganas, una y otra ejemplificadas en la oposición de mártires frente a guerreros. La forma viene dada por los paralelismos y ejemplos que ofrecen los textos bíblicos 
y las fuentes históricas relativas al momento histórico que refiere Gregorio de Tours. Así, el prólogo se limita a expresar la necesidad de que una obra histórica refiera comportamientos no ejemplarizantes junto a modelos cristianos, si bien el historiador ofrece entre líneas claves específicas, que son de género literario. Pero existen otras claves internas, propiamente textuales, que permiten profundizar en la voluntad historiográfica y, por ende, literaria que pretende plasmar el Turonense.

Gregorio de Tours recurre a Prólogos como el del Libro II para anticipar estilísticamente cómo va a ser la escritura del texto y recrear el tono formal que dicho texto ofrecerá, que, en el caso del Libro II, se organiza a partir de la plasmación de tres tipos de narraciones, la profecía, la documental o epistolográfica y, finalmente, la autobiográfica (Adamik 2012). El carácter deliberado de las opciones estilísticas aparece corroborado en el conocido epílogo-testamento que cierra la Historia Francorum (10.31), en el que Gregorio expresa irónicamente su nivel cultural a través de su aparente torpeza (como una especie de autocensura para no molestar a las elites godas) a la vez que declara que no se debe manipular ni corregir lo escrito por él, por cuanto lo ha hecho de forma voluntaria y ha sido deliberada por su parte la adopción de esa forma de escritura. De esta manera, se puede decir que es la escritura la que imprime carácter de género al contenido, si bien se hará necesario perfilar qué tipo de subgénero plantea el historiador. De ahí la existencia de diferentes niveles de redacción, lectura e interpretación del prólogo que se analiza, según se descubre en los siguientes aspectos:

a. El Turonense recurre a hipercorreciones léxicas de carácter arcaizante, las cuales remiten (dado el carácter fonético que afecta a las construcciones silábicas consonantes de tales hipercorrecciones) a la figura del historiador Salustio. Sucede con palabras como inrationabiliter y conruisse, que Gregorio de Tours podría haber desarrollado perfectamente con una grafía actualizada no sólo a su época sino a la propia época romana. Su aparición no se limita a este prólogo, sino que se descubre en otros contextos (el adverbio inrationabiliter aparece escrito siempre así en las seis ocasiones en que aparece en el conjunto de la obra); y, desde luego, no existe razón fonética alguna desde la perspectiva de la lengua vulgar que imponga tal desarrollo consonántico de sonidos en contacto sin asimilar. Se trata, por consiguiente, de una opción deliberada, que busca conferir al texto un aire venerable sin renunciar a otras connotaciones sobre el conocimiento de la lengua latina que tiene el escritor. La solución deviene más textual que propiamente oral ${ }^{3} \mathrm{y}$, subsidiariamente, permite enlazar su contenido con el de la historiografía salustiana, la cual posee, de por sí, una marcada intención política.

b. En un orden de cosas diferente, la hipercorrección fonética que acaba de ser señalada coincide con el uso de otras grafías singulares que, de manera nítida en tales casos, resultan de carácter vulgarizante (que no propiamente vulgar, por cuanto no responden a errores de evolución oral de la lengua). Se descubre en bastantes palabras recogidas en el texto, caso de filicem (que aparece hasta en quince ocasiones, inclusive como nombre propio), confusequae (enclítico equívocamente transcrito en cinco ocasiones), sullicitus (única hi-

En los términos lingüísticos que se consideran como delimitación de nuevo idioma en la definición en Wright 1989 y Banniard 1992. 
percorrección constatada, pues predomina ampliamente, hasta en diecinueve ocasiones, el uso correcto), meminiat (también como única aparición constatada), locopletavit (que se da en una segunda ocasión) u oppraesserit (en catorce ocasiones). Se trata de una forma de escritura artificial, que busca incidir en la falsa modestia de Gregorio de Tours como muestra de una supuesta falta de formación, es decir, debido a un tema tópico que, en realidad, pretende no destacar sobre el nivel lingüístico medio de la administración franca de origen no tardorromano.

c. El tercer aspecto que sobresale en el texto es la acumulación de términos bíblicos, en principio necesarios en virtud del carácter referencial que posee la literatura cristiana en su conjunto, pero, a efectos estilísticos, cuando se presentan en una especie de congeries retórica y una vez que el Libro I ya ha culminado, constituyen un síntoma preciso de una especie de recreación de la lengua bíblica a partir de topónimos y nombres propios. Así, la proliferación de palabras en las que se respeta en cierta medida el carácter extrañante de su fonética se descubre en la parte central del pasaje, casi de manera enmarcada en la doble aparición de la palabra Israheliticorum - Israheliticum: Samuhel, Fineen (con terminación de caso acusativo en n), David, Golian (acabado en n, como Fineen), Ezechie (acabado en e y no en 1), Hierusolima (con u, y no con la forma Hierosolyma; transcrito de forma incorrecta en un total de diez ocasiones a lo largo de la obra) o, finalmente, Heliseum (en equivalencia a Heliae). En definitiva, se descubre una acumulación de nombres propios en hebreo, en la que alternan las transcripciones habitualmente dadas por correctas con las irregulares, y que culminan con el relieve del tratamiento de que se hace merecedor el término Hierusolima y todo ello frente al uso gráfico establecido ya en esos momentos en la Vulgata de san Jerónimo.

d. Es posible apreciar el predominio del recurso a expresiones sinonímicas en las continuas variaciones terminológicas sobre conceptos y sintagmas semánticamente afines, como sucede con ordinem temporum y temporum series o con saeculorum ordo vel annorum ratio (con una especificación disyuntiva expresa, además del hecho de que, en relación con la palabra ratio, en líneas previas el historiador había empleado ya el adverbio inrationabiliter); el recurso se hace también patente en virtutes sanctorum y filicem beatorum vitam, en strages y excidia, o, en fin, en famis-siccitas e internitiones, por recoger las más evidentes. Se trata de una forma de recrear una lengua culta a partir de la congeries de sinónimos y a partir de variaciones semánticas que pretenden dar un aspecto literario, pero también se trata de un síntoma de lengua en marcada evolución, en la que los cambios terminológicos reflejan la duda sobre la comprensión precisa de los términos empleados. Dicho procedimiento resulta habitual en la literatura de los siglos VI y VII e incluso llega a generar obras como los Synonima de Isidoro de Sevilla en la Hispania visigótica (Elfassi 2006).

e. El párrafo se presenta rico en construcciones bimembres y quiasmáticas, con desarrollo aposicional, según aparece reflejado mediante recursos como la sinonimia, juegos léxicos o el orden de palabras, y se puede apreciar en: ordinem temporum - temporum series; mixte confusaeque; virtutes sanctorum - filicem beatorum vitam; o, por citar un último y significativo caso, strages gentium memoramus - miserorum memoremus excidia. En todas las expre- 
siones indicadas se denota un propósito elaborado, una voluntad de construcción mediante referencias internas, como un hilván que estructura no sólo el párrafo, o el libro, sino el conjunto de la obra. Se trata de un juego formal que puede equivocar sobre el sentido historiográfico al plantear polos diferentes donde no hay sino una demostración de sutileza conceptual.

f. Finalmente, en un plano específicamente literario, es posible descubrir contenidos de índole metarreferencial en relación con el tratamiento histórico. Sucede no sólo con las menciones a los textos bíblicos de carácter histórico (fundamentalmente del Libro de los Reyes, en torno al que se plantea un énfasis significativo en el pasaje), sino con las alusiones a historiadores cristianos de la talla de Eusebio y Jerónimo, autores de crónicas, $\mathrm{y}$, de forma más enfática, a Orosio, quienes constituyen una cadena de eslabones en la que el Turonense busca situar su propio texto. De cualquier forma, se trata de una mención de carácter coyuntural, por cuanto básicamente afecta a contenidos no contemporáneos a la realidad que vive Gregorio de Tours y a la que se dedica el conjunto de la Historia Francorum.

Tales reflexiones se dan también en la descripción metodológica concreta, lo cual confiere un carácter metaliterario al pasaje. Así, más allá del hecho de que las alusiones al ordo temporum y a la annorum ratio posean importantes implicaciones historiográficas relativas a la disposición propia del género de la Crónica, Gregorio de Tours anuncia que los contenidos se expondrán mixte confusequae, a la vez que se hace una mención expresa al lector a propósito de cómo tal actuación no resulta irregular, a pesar de que se mezclen asuntos cristianos y no cristianos.

\section{Un planteamiento historiográfico de carácter político}

¿Qué significa el planteamiento historiográfico descrito aplicado a una obra de la amplitud y cambios de perspectivas que ofrece la Historia Francorum? El Turonense, aunque mantiene como prioritario el decurso cronológico, permite que este se ramifique en historias paralelas, cuyo carácter es eminentemente ejemplarizante, sea en su contraste o en su acumulación. De acuerdo con ello, la expresión mixte confusequae se aplica a la realidad histórica posterior a la descrita por Orosio, aunque se sigan de fondo sus planteamientos (Martínez Pizarro 2016), y busca definir la realidad franca. En otras palabras, el historiador anticipa la convivencia de situaciones que será característica más evidente según avanza el texto, al tiempo que hace hincapié en las bases historiográficas de su manera de enfocar el relieve de los francos en la Historia Universal y de la Iglesia.

Pero hay otros aspectos referidos a la dicotomía entre motivos políticos y otros de índole distinta, sea religiosa o de actitud ética, que afectan también a la composición mixte confusequae. Se trata de la alternancia entre lo histórico y lo hagiográfico. A este respecto, Felix Thürleman (1974) ha llevado a cabo una lectura sugerente de la Historia Francorum, que gira en torno a los elementos de carácter metahistoriográfico que se pueden descubrir en el texto, en búsqueda de una definición entre los hechos históricos, los hagiográficos y las paráfrasis de tales hechos que se aprecian en prólogos y discursos insertos, con el propósito de distinguir los textos propiamente 
históricos de los expresamente hagiográficos. En este contexto, según hemos indicado, el Turonense interpreta en clave veterotestamentaria la historia de los francos (modelo que se apreciará también en historiadores posteriores como Fredegario). En realidad, en la oposición entre hechos políticos y religiosos se inpira en Agustín de Hipona a través de Orosio, según acabamos de señalar y se aprecia de forma evidente en el Prólogo al Libro II al citar de manera expresa al historiador del siglo IV.

No obstante, no hay que olvidar tampoco en relación con los propios textos bíblicos las introducciones redactadas por san Jerónimo como preámbulo a los libros del Antiguo Testamento e integradas plenamente en estos; se trata de unas introducciones que Gregorio de Tours percibe como parte de la tradición cultural del cristianismo, de forma que él mismo puede aportar en paralelo diferentes prólogos a su obra, sin menoscabo de fragmentar su texto, como no lo hace el traductor con su versión de la Vulgata. A este respecto, resulta importante destacar cómo la introducción jeronimiana a los textos bíblicos en general y a los libros del Antiguo Testamento, en el que se integran los Libros de los Reyes, en particular, se presenta también como un esfuerzo por entender la estructura del propio Antiguo Testamento en su conjunto. Sucede que en sus partes se integran como suma de elementos y figuras tanto profetas como reyes, además textos de crónicas y otros de carácter poético o ritual, en el marco de las tres partes en que se distribuye la Biblia: Pentateuco, Profetas (que incluye Reyes y Crónicas, según decimos) y Escritos Sapienciales (libros poéticos y ético-morales), algunos de los cuales Jerónimo define como hagiographa en sus Prefacios a los Libros de su versión del Antiguo Testamento. Así, de alguna manera, los libros de Reyes y Crónicas se oponen a textos cuyas figuras centrales son Job, Daniel, Ruth o Ester, entre otros, a los que se percibe más próximos a los santos cristianos; o, en otras palabras, son objeto de atención de escritos definidos como hagiographa, y, de acuerdo con ello, se les confiere mayor sentido histórico a los textos de los Profetas. Se trata de una dicotomía que puede estar presente en la percepción historiográfica del Turonense a la hora de enfocar las diferencias entre su obra hagiográfica y la Historia Francorum y también en las dicotomías que se expresan en los prólogos y, en concreto, en las que se descubren en el del Libro II.

Tal es la finalidad de la reflexión metaliteraria, de forma que el Turonense exige la complicidad de un lector que conoce los cánones habituales en las Crónicas, a los que opone las claves narrativas presentes en los libros bíblicos en las que sí tienen cabida disposiciones más aleatorias al respecto. Y no sólo los textos bíblicos. $\mathrm{Su}$ carácter ejemplar, con la mezcla de virtudes y maldades, parece inspirarse en el precedente del historiador romano Salustio, según confirma Gregorio de Tours en un pasaje del Libro IV (Hist. 4.13), en el que, arrastrado por la homonimia de un personaje con el nombre del antiguo escritor, expone:

Sed nos haec narrantis, Salustii sententiam, quam in detractaturibus historiografforum protulit, memoramus. Ait enim: Arduum videtur res gestas scribere: primum quod facta dictis exaequanda sunt; deinde quia plerique quae delecta repraehenderis malevolentia et invidia dicta putant. Sed coepta sequamur.

Podemos traducir el pasaje:

No obstante, al hacer la narración de estos hechos, evocamos aquella opinión de Salustio que expuso ante los detractores de su forma de escribir historia. Pues 
dice: «Cosa ardua parece escribir hazañas del pasado, en primer lugar, porque las palabras han de hacerse corresponder con los hechos, y, en segundo lugar, porque muchos van a considerar que los delitos que reprendas estén expuestos por aversión y envidia». Pero prosigamos con el relato emprendido.

En los dos pasajes se reiteran expresiones como la narratio, la memoria, las res gestae, entre otros. También se lleva a cabo un planteamiento historiográfico que, en equivalencia al que se da en el siglo I a. C., pretende superar la exposición de hechos aislados, meramente cronificados.

Pero es que, además, resulta fuertemente llamativo en el pasaje el uso por parte del Turonense del término delecta en lugar del delicta salustiano, una variación textual que posee evidentes consecuencias a la hora de llevar a cabo la traducción del texto y, sobre todo, la comprensión historiográfica del pasaje, que afecta al conjunto de la obra. Se trata de una variación textual que podría achacarse a un error de la tradición manuscrita, algo extraño por cuanto en, al menos, otras dos ocasiones recurre correctamente Gregorio a la palabra, en delicto (5.11) y delictum (9.41), usos que el historiador desgaja perfectamente de la raíz delect, que aparece en expresiones como ioco delectans (repetida en 3.15 y 9.20) (Tovar 2011), además de mediante otras formas verbales personales. En lo que se refiere al término verbal de raíz repraeh..., al margen de la cita de Salustio es utilizado como forma verbal únicamente en 8.20, relativa a las composiciones literarias de un obispo, cuya valoración no era unánime en cuanto a su ars, a pesar de que, en relación con el stilus había pasajes conformes en lo eclesiástico y lo religioso: Quae quibusdam quidem placuerunt, a quibusdam vero, quia artem secutus minime fuerat, repraehendebantur. Stilus tamen per loca aeclesiasticus et rationabilis erat. La oposición entre ars y stilus se establece en equivalencia con el tándem de forma y contenidos en la propuesta historiográfica, de tal manera que el hecho de que se dé cabida a motivos selectos no entra en contradicción con la finalidad instructiva de la obra.

Ello quiere decir que el uso de delecta en lugar de delicta puede ser deliberado, por cuanto, en tanto Salustio hace una caracterización ética de la historia, con un compromiso con la verdad, en Gregorio de Tours se propugna una distinción de forma: la elección de pasajes se hace de forma selectiva, a pesar de que ello no corresponda con las manifestaciones habituales del género. Por lo demás, el equívoco se documenta en Isidoro de Sevilla (Differentiae 1.D.171), donde delecta se hace equivaler con la noción de electa, o, en otras palabras, se presenta como selección deliberada, algo con lo que Gregorio de Tours busca caracterizar su obra desde una perspectiva historiográfica, según señala el historiador expresamente.

En síntesis, las ambivalencias de la historiografía cristiana se hacen presentes en las reflexiones de Gregorio de Tours en dos vertientes: de un lado, la necesidad de atenerse a una disposición cronológica de los hechos que se inicia con los textos bíblicos y a partir de aquellos libros bíblicos de carácter histórico, de ahí que recupere al final de la obra, tras el testamento literario o cultural, el numerum chronicale (10.31); de otro, la intención de aproximarse a la realidad contemporánea como una realidad sometida a debate de la misma manera que sucede en Salustio, o, por así decir, la aportación que hace la historiografía del siglo I a.C. a la tradición analística precedente. Ello resulta más significativo cuando, en realidad, Gregorio de Tours se basa en la tradición teórica agustiniana, la cual le permite la lectura cristianizada de los autores paganos, tal como el mismo Orosio había llevado a cabo. Así y en defi- 
nitiva, el Turonense reconoce en su labor una situación paralela en la que la intención ejemplarizante ha de imponerse a la meramente descriptiva a pesar de que ello provoque tanto un aparente descabalamiento en los tiempos como una interpretación equivocada sobre sus propósitos.

Ahora bien, incluso en el ámbito del desencuentro entre cronología y desarrollo de los temas, el historiador defiende el carácter como delecta (con el sentido de elaborada) de sus propuestas, si bien lo hace de forma ambigua. En efecto, el concepto de delector no parece pertenecer al mismo campo semántico que los de la malevolentia y la invidia, pues, en tanto los segundos describen una caracterización sicológica del historiador, el primero se refiere a los temas abordados de acuerdo, además, con una incorrecta transcripción de Salustio. Que tales temas se presenten como se definen en Isidoro de Sevilla, en fin, cubre el trasfondo político que subyace en la obra en su conjunto.

Es en este contexto en el que se ha de entender la expresión mixte confusequae, expresión que no aparece en el texto en más ocasiones, ni como adverbio en el caso de mixte, ni como sintagma conjunto; ciertamente, la expresión procede de Orosio, pero su finalidad historiográfica es distinta. La idea de intercalación y de simultaneidad de documentos y relatos se propone en una clave singular, característica de Gregorio de Tours: en principio el historiador presenta su propuesta como defecto retórico, cuando en realidad se trata de una manera de intervenir mediante modelos y ejemplos de actuación sobre la política contemporánea. La importancia de que aparezca la reflexión en el prólogo al Libro II, prácticamente en el engarce entre el mundo post san Martín de Tours y la aparición de los francos, y, una vez acabada la intertextualidad biblica (que justifica las obras de Eusebio y Jerónimo), revela que será el patrón dominante hasta el último libro. De esta forma, ante la descripción de la etapa histórica que llega hasta su propia época se impone la necesidad de un nuevo modelo historiográfico.

\section{Conclusión: La continuatio bíblica como subgénero historiográfico}

El debate en torno a la Historia Francorum afecta tanto a la percepción temática de la obra (a cómo avanzan los contenidos históricos desde lo universal a lo local, por ejemplo; o al tratamiento que merecen las distintas tipologías de las figuras y acontecimientos) como a su tipología genérica ${ }^{4}$. La síntesis que se desprende del análisis efectuado radica en cómo el Turonense ofrece una formulación textual que, mediante la hibridación sucesiva de intenciones (Historia Universal, Historia del mundo Franco, Historia de la Iglesia de Tours), postula una especie de continuatio bíblica: no en vano en la Biblia aparecen mezclados libros de origen, temática y tratamiento de hechos y figuras muy distintos entre sí, aunque seleccionados, sea según el modelo puntual de la crónica sea por la trascendencia ejemplar de la anécdota o la decisión.

El objetivo último de la obra está, de esta manera, puesto en la administración de los monarcas coetáneos, ante los que Gregorio de Tours presenta una especie de texto paralelo al bíblico Libro de los Reyes y, al tiempo, complementario de este. De ahí la idea de continuatio, que se complementa con la posibilidad de alternar claves históricas

Desde la exposición mediante el subgénero de la crónica al vademécum político o guide, en palabra empleada en Heinzelmann 1992. 
y hagiográficas diferentes. No obstante, el historiador relativiza el tono directo de la propuesta con una triple autocensura: una primera lingüística o sobre los límites de los conocimientos que puede poseer un representante de la población de origen galorromano; una segunda censura estilística o sobre los límites de la propia obra y, finalmente, una tercera programática o sobre los límites del género historiográfico del texto. Tal censura es intencionada: el Turonense no pretende erigirse como político por encima de unos monarcas cuya formación es, en general, ecléctica. De hecho, el escritor varía la intencionalidad y el estilo de cada época a la que se refiere en virtud de los contenidos que ofrece en los prólogos programáticos. En estos, además de consideraciones estilísticas (en apariencia vulgarizantes y con ecos bíblicos), lleva a cabo reflexiones historiográficas que, en última instancia, se pretenden con carácter ejemplar, o, en otras palabras, con una combinación de la crónica y la pragmática, pauta característica del subgénero presente en el antiguo historiador latino Salustio.

En lo que se refiere al prólogo al Libro II tales premisas estructuran el contenido del pasaje. En efecto, el Turonense propone la interpolación de sucesos cuando se respeta el orden cronológico y la amalgama de tiempos cuando conviene el contraste entre las anécdotas. A este respecto, el historiador señala que el procedimiento no sólo se da en los textos bíblicos (con ejemplos específicos de reyes del Antiguo Testamento), sino en los propios cronistas y en Orosio, toda vez que conviven hechos judíos y cristianos con realidades ajenas a la Biblia, como, fundamentalmente, las del Imperio Romano. ¿Por qué la insistencia por parte de Gregorio de Tours en tales procedimientos, si ya existen, según su propia declaración? O, con otras palabras: ¿qué aporta el Turonense a la forma historiográfica? La intencionalidad a la manera de Salustio constituye una clave indirecta, más aún cuando en sus prólogos el historiador romano juega con la dicotomía entre la racionalidad y la irracionalidad, como hace Gregorio de Tours al oponer un tándem como el que se da entre piadosos frente a malvados, sea de los grupos galorromanos y godos o de los distintos reyes francos, presentados en pares en el caso de los de mayor protagonismo (Mathisen 1993).

La respuesta se encuentra en cómo el historiador decide intervenir en la política franca de una manera eficaz y desde sus márgenes, sin que, de hecho, parezca hacerlo. Lo logra mediante un doble procedimiento: de un lado, presentar la historia franca encadenada con la bíblica; y, de otro, proponer modelos de actuación en los que alternan los aspectos negativos y los positivos, es decir, aquellos objeto de castigo y aquellos objeto de recompensa (Fournier 2020), con vistas a que los reyes adquieran perspectiva comparada en sus propias actuaciones. Así, la insistencia en la disposición cronológica responde tanto a las pautas historiográficas previas como a ese procedimiento de inserción de los francos en una línea histórica que parecía detenida con los textos de Orosio (al fin y al cabo, el Imperio Romano se incluye en los textos bíblicos). Por lo demás, la alternancia de reyes y mártires también ofrece una dicotomía sociológica (Tovar 1992), aunque la voluntad que presenta el texto de fondo es integradora, consciente el historiador de que la forma política romana no regresará ni los gobernantes godos serán desplazados. A este respecto, la aceptación del Turonense hacia la figura de Gontrán es completa, más si se le opone la figura del rey Chilperico, cuyas actuaciones detesta; mantiene el historiador así la dicotomía planteada y lleva hacia el escrito histórico las experiencias históricas coetáneas y las vivencias personales durante más de la mitad de la obra. 


\section{Referencias bibliográficas:}

Adamik, T. (2012), «Le plan de l'énontiation dans les Histoires de Grégoire de Tours», en Latin Vulgaire - Latin Tardif IX. Actes du IXe colloque international sur le Latin vulgaire et tardif, Lyon, MOM Editions, 865-879.

Banniard, M. (1978), «L'aménagement de l'histoire chez Grégoire de Tours: à propos de l'invasion de 451 (HF II 5-7)», Romanobarbarica 3, 5-38.

Banniard, M. (1992), Viva voce. Communication ecrite et communication orale du IVeme au IXemesieclesen Occident Latin, Turnhout, Brepols.

Breukelaar, A. H. B. (1994), Historiography and Episcopal Authority in Sixth-Century Gaul. The Histories of Gregory of Tours interpreted in their historical context, Göttingen, Vandenhoek \& Ruprecht.

Carozzi, C. (2007), «Grégoire de Tours historien?», en Carozzi, C. \& Taviani-Carozzi, H. (edd.), Faire l'Evénement au Moyen Age, Aix-en-Provence, Presses Universitaires de Provence, 201-218.

Croke, B. (2003), «Latin Historiography and the Barbarian Kingdoms», en Marasco, G. (ed.), Greek and Roman Historiography in Late Antiquity. Fourth to Sixth Century A.D., Leiden, Brill, 347-389.

Elfassi, J. (2006), «Genèse et originalité du Style synonymique dans les Synonyma d'Isidore de Seville», Revue des Etudes Latines 83, 225-245.

Fontaine, J. (1980), «King Sisebut's Vita Desiderii and the Political Function of Visigothic Hagiographie», en James, E. (ed.), Visigothic Spain: New Approaches, Oxford, Oxford Clarendon Press, 93-129.

Fournier, É. (2020), «Everyone but the Kings: The Rhetoric of (Non-) Persecution in Gregory of Tours' Histories», en Fournier, É. \& Mayer, W. (edd.), Heirs of Roman Persecution. Studies on a Christian and Para-christian Discourse in Late Antiquity, New York, Routledge, 184-212.

Galán Sánchez, P. J. (1994), El género historiográfico de la Crónica. Las Crónicas hispanas de época visigoda, Cáceres, Universidad de Extremadura.

Goffart, W. (1988), Narrators of Barbarian History (A.D. 550-800): Jordanes, Gregory of Tours, Bede, and Paul the Deacon, Princenton, Princenton University Press.

Halsall, G. (2007), «The Preface to Book V of Gregory of Tours' Histories: Its Form, Context, and Significance», English Historical Review 122, 297-317.

Heinzelmann, M. (1992), «Histoire, rois et prophètes. Le rôle des éléments autobiographiques dans les Histoires de Grégoire de Tours: une guide episcopal à l'usage du roi chrétien», en De Tertullien aux Mozarabes. Mélanges offerts à Jacques Fontaine I, Paris, Institute d'Études Augustiniennes, 537-550.

Heinzelmann, M. (2001), Gregory of Tours. History and Society in the Sixth Century, Cambridge, Cambridge University Press.

Heinzelmann, M. (2015), «The Works of Gregory of Tours and Patristic Tradition», en Murray, A. C. (ed.), A Companion to Gregory of Tours, Leiden, Brill, 279-336.

Herrera Roldán, P. (2013), Gregorio de Tours. Historias, Cáceres, Universidad de Extremadura.

Janson, T. (1964), Latin Prose Prefaces. Studies in Literary Conventions, Stockholm, Almqvist \& Wiksell.

Krusch, B., \& Levinson, W. (1951, reed.), Historiarum Libri Decem. Berlin, Monumenta Germaniae Historica - Scriptores Rerum Merovingicarum. 
Martínez Pizarro, J. (2016), «Gregory of Tours and the Literary Imagination: Genre, Narrative Style, Sources, and Models in the Histories», en Murray, A. C. (ed.), A Companion to Gregory of Tours, Leiden, Brill, 337-374.

Mathisen, R. W. (1993), Roman aristocrats in barbarian Gaul: strategies for survival in an age of transition, Austin, University of Texas Press.

Meens, R., et al. (edd.) (2016), Religious Franks: Religion and Power in the Frankish Kingdoms. Studies in Honour of Mayke de Jong, Manchester, Manchester University Press.

Mitchell, K., \& Wood, I. (edd.) (2002), The World of Gregory of Tours, Leiden, Brill.

Moore, M. E. (2011), A Sacred Kingdom: Bishops and the Rise of Frankish Kingship, 300850, Washington, Catholic University of American Press.

Murray, A. C. (2005), Gregory of Tours. The Merovingians, Peterborough (Canadá), Broadview Press.

Murray, A. C. (2008), «Chronology and the Composition of the Histories of Gregory of Tours», Journal of Late Antiquity 1, 157-196.

Murray, A. C. (2015), «The Composition of the Histories of Gregory of Tours and Its Bearing on the Political Narrative», en Murray, A. C. (ed.), A Companion to Gregory of Tours, Leiden, Brill, 61-101.

Oldoni, M. (1972), «Gregorio di Tours e i Libri Historiarum, letture e fonti, metodi e ragioni», StudiMedievali 13, 563-700.

Reimitz, H. (2015), «The Early Medieval Editions of Gregory of Tours' Histories», en Murray, A. C. (ed.), A Companion to Gregory of Tours, Leiden, Brill, 517-565.

Thürlemann, F. (1974), Der historische Diskurs bei Gregor von Tours: Topoi und Wirklichkeit, Bern, Peter Lang Publishing.

Tovar Paz, F. J. (1992), «Sentido de las Passiones hispánicas sub Datiano praeside» en Alvar, J., Blánquez Pérez, C. \& García Wagner, C. (edd.), Héroes, semidioses y daimones, Madrid, Ediciones Clásicas, 433-462.

Tovar Paz, F. J. (2011), «Sentido del humor e ironía en la Historia Francorum, de Gregorio de Tours», Myrtia 26, 105-121.

Wallace-Hadrill, J. M. (2019), The Long-Haired Kings. And Other Studies in Frankish History, London, Routledge.

Werner, K.-F. (1987), «Gott, Herrscher und Historiograph. Der Geschichtsschreiber als Interpret des Wirkens Gottes in der Welt und Ratgeber der Könige (4-12 Jahrhundert)», en Hehl, E. D., Seibert, H. \& Staab, F. (edd.), Deus qui mutat tempora. Menschen und Institutionem im Wandel des Mittelaters. Festschrift für Alfons Becker zu seinem $65 \mathrm{Ge}$ burtstag, Sigmaringen, Thorbecke, 1-31.

Wood, I. N. (1994), Gregory of Tours, Oxford, Headstar History Papers.

Wright, R. (1989), Latín tardio y romance temprano, Madrid, Gredos. 